\title{
Optimal Design of Ultrasonic Transducer System Applied on Biolipid Extraction from Chlorella
}

\author{
Han-Sung Huang, Shao-Yi Hsia, ${ }^{*}$ and Shiuh-Kuang Yang ${ }^{2}$ \\ Graduate Institute of Engineering Science and Technology, \\ National Kaohsiung First University of Science and Technology, Kaohsiung City, Taiwan, R.O.C. \\ ${ }^{1}$ Department of Mechanical \& Automation Engineering, Kao-Yuan University, \\ Kaohsiung City, 82151, Taiwan, R.O.C. \\ ${ }^{2}$ Department of Mechanical and Electro-Mechanical Engineering, National Sun Yat-sen University, \\ Kaohsiung City, 80424, Taiwan, R.O.C.
}

(Received August 31, 2015; accepted March 1, 2016)

Keywords: ultrasonic transducer, biodiesel, extraction, biomechatronics

Algae have the ability to convert solar energy into organic compounds through photosynthesis and store them as lipids. The final step is the conversion of biolipids into biodiesel, which is a way to reduce carbon dioxide emissions and which can be considered as an inexhaustible alternative energy source. The process of studying the effects of biomechatronics involves an ultrasonic transducer and light emitting diode (LED) lights, which affect algal lipids. In an optimum extraction experiment, $0.18 \mathrm{~g}$ lipid can be extracted per gram of Chlorella after $5 \mathrm{~d}$. Experiments with ultrasound increased lipid extraction by $19.13 \%$ compared with controls. The results of this study can be used for increasing the amount of lipid production that can be extracted from Chlorella, contributing to the development of better cultivation techniques with adequate environmental protection.

\section{Introduction}

Raw materials for biodiesel production are currently extracted from plants as oils, such as soybean oil, rapeseed oil, and palm oil. Being edible, these oils are restricted in supply, so it is necessary to look for sustainable and stable sources of oils to satisfy the large demand. Microalgae are a feasible option as a biodiesel source because they fix atmospheric carbon dioxide via photosynthesis and manufacture oils similar to other plants. Generally speaking, the oil production rate of microalgae is faster than in terrestrial plants, offering competitiveness in both economics and time. Microalgae reveal distinct oil contents, depending on the species, and many microalgal taxa do not produce oil. The oil content of typical oil-producing microalgae is about $20-50 \%$ by weight. Since the oil production rate of microalgae depends on their growth rate, species with high oil production rates are the first choices for research.

The use of oil produced from microalgae as a biodiesel source was first proposed in 1980, but it was not emphasized. It was not until the relatively recent rise in crude oil prices that the development of renewable biodiesel using microalgae was broadly studied. Many industrial, governmental, and university research units realize the potential to produce biodiesel with

"Corresponding author: e-mail: syhsia@cc.kyu.edu.tw 
microalgae to replace diesel fuel from fossil fuel.(1) Relevant research indicates that cultivating microalgae on one hectare of land could produce up to 100 tons of oil annually, which is far higher than oil production in other plants. The robustness of producing oil with microalgae is now apparent. The cost of producing biodiesel with microalgae is still higher than fossil diesel; however, microalgal biodiesel presents a potential to replace fossil diesel as crude oil prices continuously rise and the cultivation of microalgae is constantly improved. In summary, it is an important challenge to develop bioenergy by reducing the cost of producing bio-oil with microalgae. Future efforts to improve the process and the use of genetic engineering are expected to enhance the properties of photobioreactors and the oil production rate of microalgae as well as establish a sustainable and environmentally sound renewable energy.

Spolaore et al. (2005) (2) discussed the first utilization of microalgae in China more than 2000 years ago, when fat choy was eaten during famine. However, microalgae biotechnology was not actually developed until the last century, and now there are many commercial applications. For instance, microalgae play a critical role in aquaculture, and their cellular compounds are used in human food, animal feed, and cosmetics. Coakley and Hampton ${ }^{(3)}$ discovered that cavitation produced ultrasonically at a frequency of $1 \mathrm{MHz}$ and intensity of $515 \mathrm{~W} / \mathrm{cm}^{2}$ decreased the number of amoeba cells. In research with ultrasound and lasers, Rajasekhar et al. ${ }^{(4)}$ found that the poisonous Spirulina and Microcystis aeruginosa exposed in a lagoon to $20 \mathrm{kHz}$ of ultrasound exhibited reduced growth rates, with the highest decrement appearing in the initial $5 \mathrm{~min}$. Under the same ultrasound conditions, the growth of three types of microalgae was restrained in the order Circinalis $>$ Microcystis aeruginosa $>$ Chlorella. Ultrasound selectively eliminates harmful Spirulina from the algal community in a lagoon. Hsia et al. ${ }^{(5)}$ studied the combination of mechanical and biological effects from ultrasound that was used to increase bio-hydrogen production from dark-fermentation bacteria. In this study, four controlling factors (ultrasonic frequency, energy, exposure time, and starch concentration) were considered in calculating the highest hydrogen production using the Taguchi method. Under the best operating conditions, the biohydrogen production efficiency of dark-fermentation increased $19.11 \%$. Hsia and $\mathrm{Chou}^{(6)}$ also discussed enhancing algal growth by stimulation with light emitting diode (LED) lighting and ultrasound. This report describes our research in indoor algae production using LED lights and ultrasound and manipulating other growth factors at different temperatures. Ultrasound treatment at the alga's natural resonant frequency was varied to determine optimal algal growth using the Taguchi method to plan and analyze experiments. The results were very satisfying, showing an $8.23 \%$ increase in the growth rate by the fifth day due to ultrasound treatment and an amazing $27.01 \%$ increase in growth rate due to biomechanical stimulation.

Regarding research on extraction, Chisti et al.(7) pointed out that fossil fuel is unsuitable for general use. Fossil fuel generates large amounts of carbon dioxide, so bioenergy is a more suitable substitute. Planting oilseed crops on limited land cannot meet demand; therefore, algae are considered favorable organisms for producing bioenergy. Besides, many microalgae have higher oil production efficiencies than oilseed crops. Gao et al. ${ }^{(8)}$ utilized ultrasound to enhance the supercritical $\mathrm{CO}_{2}$ extraction of lutein ester in Tagetes erecta and discovered that it enhanced the solid-phase mass transfer coefficient to produce the same amount of lutein ester with lowpressure and low-temperature extraction. Their experimental results revealed that the optimal size of Tagetes erecta is $0.245-0.350 \mathrm{~mm}$, extraction pressure $35 \mathrm{MPa}$, temperature $55^{\circ} \mathrm{C}, \mathrm{CO}_{2}$ flow velocity $10 \mathrm{~kg} / \mathrm{h}$ lasting for $3 \mathrm{~h}$, exposure power $400 \mathrm{~W}$, exposure frequency $25 \mathrm{kHz}$, and cycle of exposure $6 \mathrm{~s} /$ rest $9 \mathrm{~s}$. In the same year, Converti et al. ${ }^{(9)}$ studied the effects on the production of 
biodiesel of extracting oil from Nannochloris oculata and Chlorella by controlling temperature and nitrogen concentration and using quantitative and qualitative gas chromatography to analyze the oil. Experimental results showed that the oil content increased from 7.90 to $14.92 \%$ when the temperature was increased from 20 to $25^{\circ} \mathrm{C}$, oil content decreased from 14.71 to $5.90 \%$ when the temperature was increased from 25 to $30{ }^{\circ} \mathrm{C}$, and optimal growth occurred when nitrogen content was reduced by $75 \%$. The oil content of Nannochloris oculata increased from 7.90 to $15.31 \%$ and the oil content of Chlorella increased from 5.90 to $16.41 \%$.

Optimal oil production was not discussed in the cited research. For this reason, freshwater Chlorella sp. purchased from Tungkang Biotechnology Research Center was used to investigate mechanical and biological effects on oil production. Figure 1 shows a schematic diagram of biolipid extraction from Chlorella using LEDs and ultrasound stimulation, and the experimental flowchart is noted in Fig. 2. In this study, the Taguchi method was used to find the optimal combination for enhancing Chlorella oil production and contributing to environmental protection.

\section{Theory}

\subsection{Introduction of Chlorella}

The microorganism Chlorella is found in salt- and freshwater and is a large genus of algae in the Charophyta. They form a paraphyletic group within the kingdoms Plantae or Protista. Chlorella, as unicellular or clustered organisms, has two flagella per cell. Most of the 6000 species are unicellular, but a few form clusters or strip filaments. Chlorella cells have spheroidal or oval shapes depending on the species, have a diameter of 2-12 $\mu \mathrm{m}$, and exhibit different frond sizes according to species, frond production time, nutrition, and environment. Chlorella consists of $40 \%$ protein, $10 \%$ fat, $10 \%$ carbohydrate, $6 \%$ ash, and $34 \%$ chlorophyll.

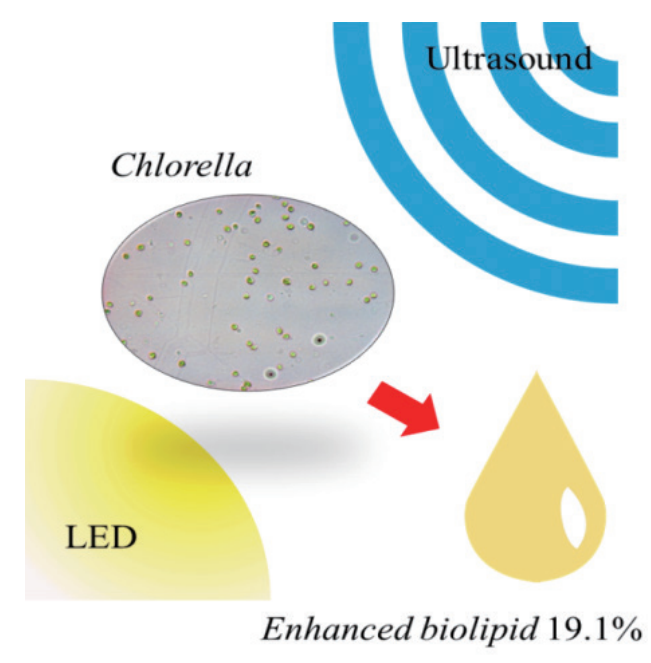

Fig. 1. (Color online) Schematic of biolipid extraction by stimulation with LEDs and ultrasound.

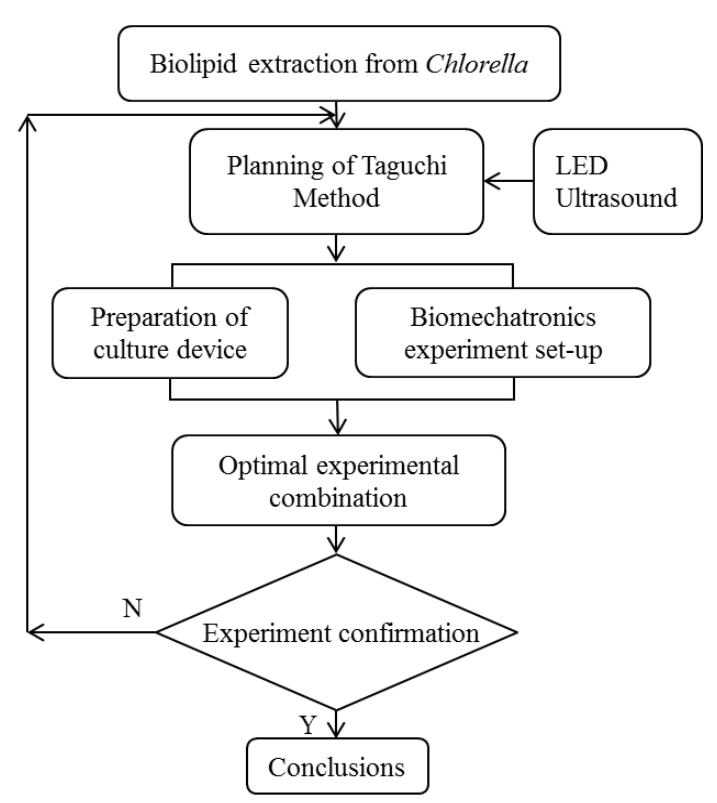

Fig. 2. Flowchart of algal growth experiment. 


\subsection{Culture media}

Culture media generally contain a carbon source, nitrogen source, inorganic salts, and microelements. A carbon source provides the carbon required for growth and reproduction. Either organic or inorganic nitrogen was used as a source in this research. There are nine major elements required: carbon, hydrogen, oxygen, nitrogen, phosphorus, potassium, sulfur, calcium, and magnesium. The microelements boron, manganese, zinc, molybdenum, cobalt, iodine, and copper are also required by Chlorella. The culturing fertilizer used in our experiments was Walne's medium. ${ }^{(6)}$

\subsection{LED application}

Progress in electro-optical technology has enhanced the brightness and efficiency of LEDs such that these light sources are feasible for use in agricultural production. LEDs have high photovoltaic conversion efficiencies, small volumes, long service lives, fixed wavelengths, and low heat production, and use only direct current. These advantages offer adjustable light intensity, adjustable light quality (percentages of red/blue or red/far-infrared), low cooling load, and the ability to enhance production per unit area in comparison with currently used systems that rely on fluorescent lamps or high-pressure sodium lamps as artificial light sources. As a result, LEDs are a suitable artificial light source for indoor and controlled agricultural production environments (e.g., plant tissue culture and plant growth chambers).

\subsection{Biological effects of ultrasonic transducer systems}

Many studies show that ultrasound transmits energy via particle vibrations within propagation media to form an ultrasound field. When ultrasound irradiates biological media at a distinct frequency and intensity, various physical effects appear in ultrasound energy and particles. These are divided into thermal and nonthermal effects and then are further divided into mechanical and cavitation effects. Cavitation effects are regarded as nonthermal and have the greatest impact on biological tissue, as ultrasound transmits a wave of condensation and rarefaction in liquids that results in cavitation. Cavitation happens when a liquid generates tiny bubbles owing to the positive and negative pressures of a moving ultrasound wave, or tiny bubbles occur in the liquid owing to ultrasound vibration, growth, contraction, or crashing. When cells experience the high shear wave that is generated from vibrating bubbles or bubble crashing, a series of biological reactions occurs. In this study, a natural frequency was used in the ultrasound exposure experiments by setting a different pulse intensity for microorganism vibration. ${ }^{(5,6)}$

\subsection{Extraction}

Extraction separates different substances dissolved in a solution by taking advantage of the distinct solubility of each substance in a solvent. There are two types of extraction. The first is liquid-liquid extraction, which uses a solvent to extract target substances within a liquid mixture. Target substances should not dissociate within the extracted liquid mixture and should present selective dissolution, favorable thermal and chemical stabilities, and low toxicity and corrosivity (e.g., separating phenol from coal tar with benzene and alkene from petroleum distillates with organic 
solvents). The second type, called solid-liquid extraction or leaching, separates the contents in a solid mixture with solvent, as is done by leaching carbohydrates in beetroot with water, leaching soybean oil with alcohol to enhance oil production, and leaching the effective contents of traditional Chinese medicine with water to produce a fluid extract called a "leachate". Although extraction is often used in chemical experiments, the process does not change the chemical content of the extracted material (or chemical reaction), as extraction is a physical process.

\section{Experimental Equipment and Procedures}

The effects of ultrasonic exposure on oil production from freshwater Chlorella were explored in this experiment, in which freshwater Chlorella was produced and analyzed for optimal combination with Hyponex. The electromechanical effects of ultrasonic exposure and LED exposure are also discussed. Figure 3 represents the instrument layout of the ultrasonic transducer and LED light system, and the Chlorella produced was further extracted for bio-oil.

\subsection{Selected parameters in the ultrasonic transducer system}

If an ultrasonic transducer is used in an immersion system, it can be represented as a terminated two-port system as shown in Fig. 4. The acoustic port is always terminated, i.e., the output force and velocity are related to one another.

For an acoustic wave radiating into a fluid $p(x, \omega)$ on the face of the acoustic output port of the transducer is given in terms of the uniform normal velocity $v_{\mathrm{t}}(\omega)$ at that port by the RayleighSommerfeld integral:

$$
p(\boldsymbol{x}, \omega)=\frac{-i \omega \rho v_{\mathrm{t}}(\omega)}{2 \pi} \int_{\mathrm{S}} \frac{\exp (i k r)}{r} d S(\boldsymbol{y}),
$$

where $\boldsymbol{x}$ and $\boldsymbol{y}$ are two points on the surface, $S$ is the transducer face, $\rho$ is the density of the fluid, $k$

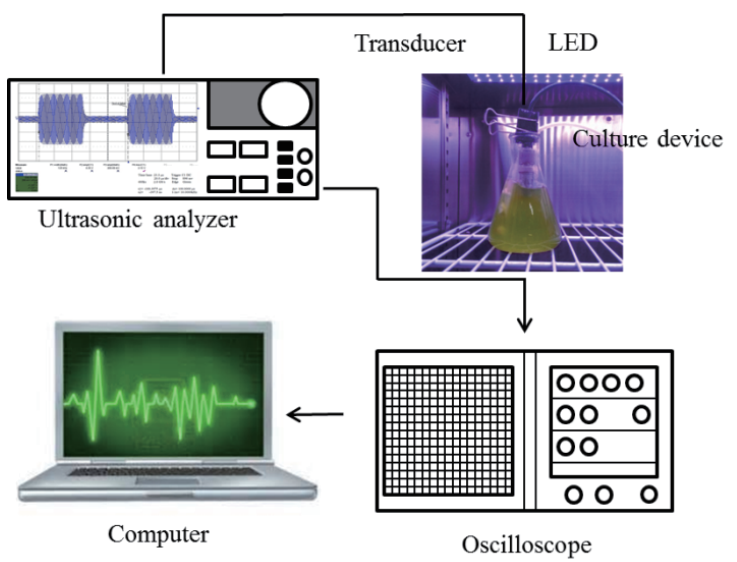

Fig. 3. (Color online) Layout of the ultrasonic transducer and LED light system.

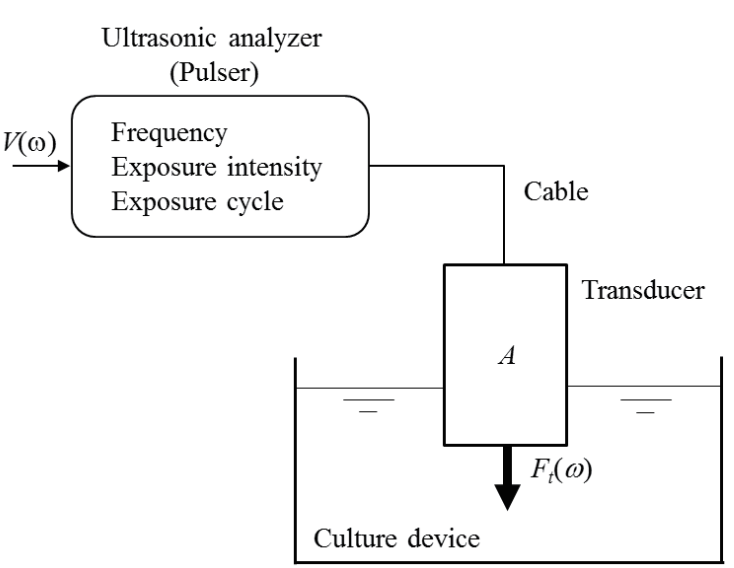

Fig. 4. A simplified diagram of an immersed ultrasonic transducer radiating into a fluid. 
is the wave number for waves propagating in the fluid, and $r=|\boldsymbol{x} \times \boldsymbol{y}|$ is the distance between $\boldsymbol{x}$ and $\boldsymbol{y}$. Since the compressive force, $F_{t}$, at the transducer's output port is just the integral of this pressure, we have

$$
\begin{aligned}
F_{\mathrm{t}}(\omega) & =\left[\frac{-i \omega \rho}{2 \pi} \int_{\mathrm{S}} \int_{\mathrm{S}} \frac{\exp (i k r)}{r} d S(\boldsymbol{y}) d S(\boldsymbol{x})\right] v_{\mathrm{t}}(\omega) \\
& =Z_{r}^{a}(\omega) v_{\mathrm{t}}(\omega),
\end{aligned}
$$

where the term in brackets in Eq. (2), $Z_{r}^{a}$, is called the transducer radiation impedance. Greenspan ${ }^{(10)}$ has shown that the double integrals in Eq. (2) can be performed for a circular planar piston transducer of radius $a$. For most ultrasonic transducers, for example, at $1 \mathrm{MHz}$, a $12.5 \mathrm{~mm}$ radius piston transducer radiating into water has a $k a$ value of approximately 53. Thus, even though such ultrasonic transducers generate sound beams that are not just plane waves, their acoustic radiation impedances can generally be taken as simply as the constant value of a plane wave.

Meanwhile, we know that the experimental system is connected to the pulser, cabling, and transducer models in an ultrasonic immersion system (Fig. 4). This equivalent circuit model is shown schematically in Fig. 5. In this case, $V, I$, and $Z$ are the driving voltage, current, and electrical impedance, respectively. The subscript " $i$ " denotes the pulser's electrical port, and "in", the transducer's electrical port. The symbol $(T)$ is the transfer matrix of the cabling between the pulser and the transmitting transducer. ${ }^{(11)}$ Figures 4 and 5 also indicate that three parametersfrequency, exposure intensity, and exposure cycle - could be used in this study. Hence, the electromechanical effects of ultrasound exposure on Chlorella growth were investigated to determine the differences caused in oil production by natural and non-natural ultrasound frequencies, different acoustic intensities, and distinct exposure times. Frequency was calculated with Rayleigh-Plesset equations, ${ }^{(12)}$ revealing the natural frequency of Chlorella to be in the range of $0.5-3.5 \mathrm{MHz}$. A monocrystalline straight-beam longitudinal-wave immersion probe with resonance frequencies of 0.5 and $1 \mathrm{MHz}$ was selected as the natural frequency exposure probe, and a $5 \mathrm{MHz}$ non-natural frequency probe was used for comparison. A power amplifier was used to amplify the modulated wave from the signal generator to control the excited ultrasound intensity of the probe for parameter control. $10 \mathrm{kHz}$ square waves and sinusoidal waves were combined in the experiment as the modulated waves displayed on the computer screen in Fig. 3. Based on the irradiation frequency of the transducer, the sinusoidal wave frequency was adjusted to 0.5 and 1

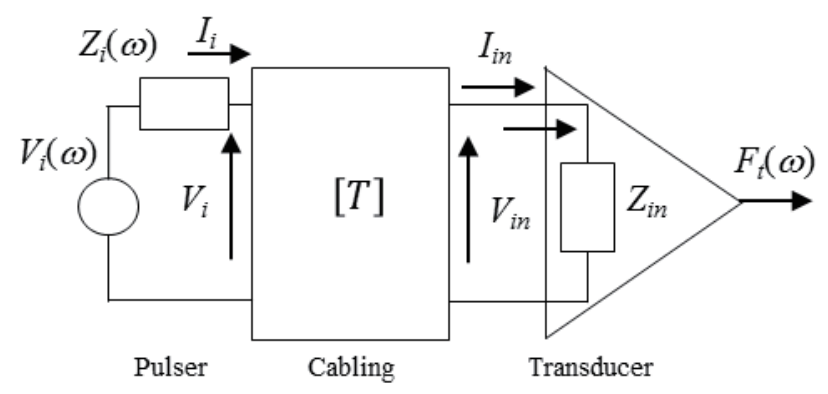

Fig. 5. The equivalent circuit model for the pulser, the cabling, and the transmitting transducer. 
MHz. Sound energy density values of $1.24,2.01$, and $2.75 \mathrm{~mW} / \mathrm{cm}^{2}$ were measured at a distinct frequency that it is used to explore the effects of exposure frequency with intensity on Chlorella growth. The effects of an ultrasound exposure cycle on Chlorella have not been definitively discussed in the literature. Ultrasound cycles of exposure $5 \mathrm{~min} /$ rest $5 \mathrm{~min}$, exposure $10 \mathrm{~min} /$ rest 10 $\mathrm{min}$, and exposure $15 \mathrm{~min} /$ rest $15 \mathrm{~min}$ were applied in our experiments.

\subsection{LED exposure experiment}

The photoelectric effects of LED exposure were also studied to determine the effects of optical wavelength, optical intensity, and exposure cycle on oil production. Blue light (456 nm) and red light $(630 \mathrm{~nm})$, in addition to white light, were used, as red is known as the light of growth and blue releases the parasympathetic system, which might affect Chlorella. The absorption peaks of chlorophyll photosynthesis are mainly red and blue bands, which are difficult for traditional lamps to produce but are easily achieved by LEDs. Photosynthetic rates increase when optical intensity is enhanced under certain conditions until the most suitable intensity is reached, after which photosynthetic rates gradually decrease. Little research has been devoted to the effects of LED exposure cycle on Chlorella. The effects of cycle frequency on Chlorella growth during the day and night were studied in this experiment by setting cycles to exposure $3 \mathrm{~h} /$ rest $3 \mathrm{~h}$, exposure $6 \mathrm{~h} /$ rest $6 \mathrm{~h}$, and exposure $12 \mathrm{~h} /$ rest $12 \mathrm{~h}$.

\subsection{Temperature}

Temperature is a critical factor in Chlorella growth. In an appropriate environment, increasing the temperature accelerates Chlorella growth and metabolism as well as reproduction. Nevertheless, there is an upper limit for survival. Chlorella growth is restrained, resulting in aging, when the temperature is too high, while reproduction in Chlorella is reduced and without fission when the temperature is too low. At low temperatures, Chlorella's absorption of nutrients is reduced. Accordingly, the selection of temperature in the Chlorella growth process is extremely important. Previous research ${ }^{(5)}$ reveals that the optimal growth rate of Chlorella sp. is at temperatures $<25{ }^{\circ} \mathrm{C}$, so the temperature was set at $25{ }^{\circ} \mathrm{C}$ in our experiments.

\subsection{Taguchi Method planning}

The variable parameters discussed were combined in this study and the Taguchi method orthogonal array was utilized for experiment planning (Table 1). Three replicates of each experiment were carried out. An analysis of variance was done to learn the key factors in the experiment and to determine the optimal combination for the Chlorella experiment.

\subsection{Extraction experiment}

The extraction procedure in this experiment was carried out as follows:

(1) Chlorella in a $50 \mathrm{ml}$ centrifuge tube was placed in an ultracentrifuge for $15 \mathrm{~min}$ at $4000 \mathrm{rpm}$; a precise electronic balance was used to measure the weight of the empty small glass bottle (without the lid). 
Table 1

Six factors and two/three levels of the orthogonal array $L_{12}\left(2^{1} \times 3^{5}\right)$.

\begin{tabular}{|c|c|c|c|c|c|c|}
\hline \multirow[b]{3}{*}{ Exp. } & A & $\mathrm{B}$ & $\mathrm{C}$ & $\mathrm{D}$ & $\mathrm{E}$ & $\mathrm{F}$ \\
\hline & \multicolumn{3}{|c|}{ LED } & \multicolumn{3}{|c|}{ Ultrasound } \\
\hline & $\begin{array}{c}\text { Exposure } \\
\text { intensity (lux) }\end{array}$ & $\begin{array}{l}\text { Wave length } \\
(\mathrm{mm})\end{array}$ & $\begin{array}{l}\text { Exposure } \\
\text { cycle }(\mathrm{h})\end{array}$ & $\begin{array}{l}\text { Frequency } \\
(\mathrm{MHz})\end{array}$ & $\begin{array}{l}\text { Exposure } \\
\text { intensity } \\
\left(\mathrm{mW} / \mathrm{cm}^{2}\right)\end{array}$ & $\begin{array}{l}\text { Exposure } \\
\text { cycle (min) }\end{array}$ \\
\hline 1 & 8000 & Blue (456) & $3 / 3$ & 0.5 & 1.24 & $5 / 5$ \\
\hline 2 & 8000 & Blue (456) & $6 / 6$ & 1 & 2.75 & $15 / 15$ \\
\hline 3 & 8000 & White & $3 / 3$ & 5 & 2.01 & $15 / 15$ \\
\hline 4 & 8000 & White & $12 / 12$ & 0.5 & 2.75 & $10 / 10$ \\
\hline 5 & 8000 & Red (630) & $12 / 12$ & 1 & 2.01 & $5 / 5$ \\
\hline 6 & 8000 & Red (630) & $6 / 6$ & 5 & 1.24 & $10 / 10$ \\
\hline 7 & 13000 & Red (630) & $6 / 6$ & 0.5 & 2.75 & $5 / 5$ \\
\hline 8 & 13000 & Red (630) & $3 / 3$ & 1 & 1.24 & $15 / 15$ \\
\hline 9 & 13000 & White & $6 / 6$ & 1 & 2.01 & $10 / 10$ \\
\hline 10 & 13000 & White & $12 / 12$ & 5 & 1.24 & $5 / 5$ \\
\hline 11 & 13000 & Blue (456) & $12 / 12$ & 0.5 & 2.01 & $15 / 15$ \\
\hline 12 & 13000 & Blue (456) & $3 / 3$ & 5 & 2.75 & $10 / 10$ \\
\hline
\end{tabular}

(2) A glass dropper was used to remove the water in the centrifuge tube, Chlorella was washed into the small empty bottle with methanol, and a rotary evaporator and a freeze dryer were used to remove the methanol and water.

(3) A precise electronic balance was used to measure the weight of the small glass bottle (without the lid), which was then deducted from the weight of the empty bottle in order to determine the weight of the dry algae.

(4) Chlorella was then mixed into a 2:1 methanol-to-chloroform solvent. Filter paper was placed in a short-stem funnel to transfer the Chlorella to an eggplant-type flask. This mixture was oscillated ultrasonically for $30 \mathrm{~min}$ and, after reduction in a rotary evaporator, the amount was poured into a small glass bottle. The rotary evaporator and freeze dryer were used again to remove methanol and chloroform.

(5) Chlorella was mixed in an organic solvent containing methanol:chloroform:1\% $\mathrm{NaCl}$ (2:2:1 ratio) and placed in a separatory funnel. The solubility of oil is different from other components, so the bottom layer of the solution was removed and placed in an eggplant-type flask, and the volume was reduced using a rotary evaporator and poured into a small glass bottle.

(6) After removal of solvent with a rotary evaporator and a freeze dryer, a precise electronic balance was used to measure the weight of the small glass bottle (without the lid), which was deducted from the previously measured weight of the full bottle to acquire the weight of the oil.

\section{Results and Discussion}

The production of freshwater Chlorella was analyzed. A growth curve was drawn based on the data and various control factors, and levels were judged with an analysis of variance after the Taguchi method orthogonal array experiment to find out the optimal combination. 


\subsection{Oil extraction analysis}

The aim of this experiment was to enhance oil production; i.e., to consider quality characteristics. The Chlorella was weighed after centrifuging in a $50 \mathrm{~cm}^{3}$ centrifuge bottle and dried to determine the relationship between freshwater Chlorella concentration and dry weight (Fig. 6). The $x$-axis is the freshwater Chlorella concentration, the $y$-axis is Chlorella dry weight, and $R^{2}$ in the formula is the exact value of linearity, where $R^{2}$ values closer to 1 indicate better curve fitting. In biological and agricultural research, $R^{2}$ values $>0.9$ are acceptable. Dried freshwater Chlorella was then extracted for its oil (Fig. 7). Freshwater Chlorella production and extraction rates are shown in Table 2. Since an estimate of maximum oil production was the ultimate objective of this study, freshwater Chlorella production was multiplied by the oil extraction rate to determine extractable oil production.

Formulas from the Taguchi method were used to calculate the factor effect table of quality characteristics of the oil extraction rate (Table 2). In this method, quantified experimental results are noted quality characteristics and can achieve an ideal by determining the control factors in the experimental design.(6) The factor effect diagram is shown in Fig. 8, in which the horizontal axis represents control factors and levels and the vertical axis is the oil extraction rate. The optimal oil extraction rate combination was ranked as A2, B1, C2, D3, E3, and F2, meaning that the optimal effect appeared with an LED intensity of 13000 lux, LED blue wavelength (456 nm), LED cycle of exposure $6 \mathrm{~h} /$ rest $6 \mathrm{~h}$, ultrasound frequency of $5 \mathrm{MHz}$, ultrasound intensity of $2.75 \mathrm{~mW} / \mathrm{cm}^{2}$,



Fig. 6. (Color online) Relationship between freshwater Chlorella concentration and dry weight in $50 \mathrm{~cm}^{3}$.
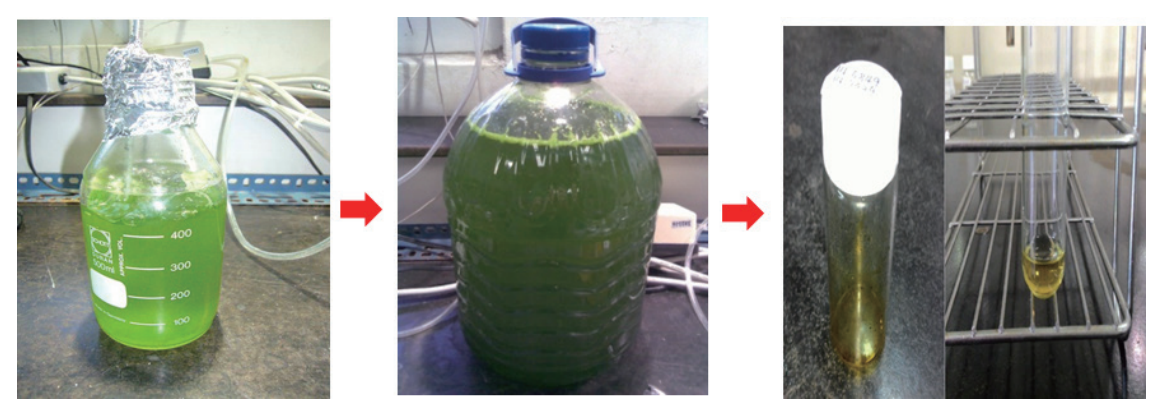

Fig. 7. (Color online) Freshwater Chlorella was cultivated and then its oil extracted. 
Table 2

Quality characteristics at different biolipid extraction rates.

\begin{tabular}{lcccrrr}
\hline $\begin{array}{l}\text { Quality characteristics of } \\
\text { oil extraction rate (\%) }\end{array}$ & $\mathrm{A}$ & $\mathrm{B}$ & $\mathrm{C}$ & $\mathrm{D}$ & $\mathrm{E}$ & $\mathrm{F}$ \\
\hline Level 1 & 10.60 & 11.63 & 10.43 & 9.25 & 9.70 & 9.55 \\
Level 2 & 10.72 & 10.35 & 12.05 & 11.00 & 10.65 & 11.43 \\
Level 3 & - & 10.00 & 9.50 & 11.73 & 11.63 & 11.00 \\
Effect & 0.12 & 1.63 & 2.55 & 2.48 & 1.93 & 1.88 \\
Rank & 6 & 5 & 1 & 2 & 3 & 3 \\
\hline
\end{tabular}



Fig. 8. (Color online) Factor effect diagram of quality characteristics of the oil extraction rate.

and ultrasound cycle of exposure $10 \mathrm{~min} /$ rest $10 \mathrm{~min}$. The LED cycle was regarded as the primary factor, followed by ultrasound frequency, ultrasound intensity, ultrasound cycle, and LED intensity. The factor effect table of quality characteristics of oil production by extraction is shown in Table 3, and the factor effect diagram is shown in Fig. 9, in which the horizontal axis represents control factors and levels and the vertical axis represents oil production.

The optimal combination of oil production by extraction was ranked as A2, B3, C2, D3, E3, and F2, meaning that the optimal effect appeared with an LED intensity of 13000 lux, LED wavelength red $(630 \mathrm{~nm})$, LED cycle of exposure $6 \mathrm{~h} /$ rest $6 \mathrm{~h}$, ultrasound frequency of $5 \mathrm{MHz}$, ultrasound intensity of $2.75 \mathrm{~mW} / \mathrm{cm}^{2}$, and ultrasound cycle of exposure $10 \mathrm{~min} /$ rest $10 \mathrm{~min}$. LED intensity was considered the most important factor, followed by LED cycle, ultrasound cycle, ultrasound frequency, LED wavelength, and ultrasound intensity.

\subsection{Experimental confirmation of optimal oil extraction}

To confirm the optimal combination, additional formulas were first used to calculate the freshwater Chlorella extraction rate and production as well as predict optimization after $5 \mathrm{~d}$ of ultrasound exposure. The optimal oil extraction rate after an ultrasound exposure of $5 \mathrm{~d}$ increased $2.80 \%$, and the relative value increased $24.34 \%$. Optimization after adding ultrasound enhanced the extract content of oil extraction production by $0.60 \mathrm{mg}$, and the relative value increased $18.63 \%$ (Table 4). The confirmation experiment further revealed that the oil extraction rate could be absolutely enhanced $2.90 \%$ after an ultrasound exposure of $5 \mathrm{~d}$, and relative oil extraction efficiency increased $18.83 \%$; the absolute extract content of oil extraction production with 
Table 3

Quality characteristics for different biolipid production by extraction.

\begin{tabular}{lcccccc}
\hline $\begin{array}{l}\text { Quality characteristics of } \\
\text { oil extraction rate (\%) }\end{array}$ & $\mathrm{A}$ & $\mathrm{B}$ & $\mathrm{C}$ & $\mathrm{D}$ & $\mathrm{E}$ & $\mathrm{F}$ \\
\hline Level 1 & 1.22 & 1.63 & 1.54 & 1.54 & 1.82 & 1.87 \\
Level 2 & 2.42 & 1.69 & 2.30 & 1.86 & 1.75 & 2.10 \\
Level 3 & - & 2.14 & 1.62 & 2.07 & 1.89 & 1.48 \\
Effect & 1.20 & 0.50 & 0.76 & 0.53 & 0.14 & 0.62 \\
Rank & 1 & 5 & 2 & 4 & 6 & 3 \\
\hline
\end{tabular}



Fig. 9. (Color online) Factor effect diagram of quality characteristics of oil production by extraction.

Table 4

Prediction of additional formulas with and without ultrasound treatments.

\begin{tabular}{lcccc}
\hline \multirow{2}{*}{ Confirmatory experiment } & Without ultrasound & With ultrasound & \multicolumn{2}{c}{ Raised content and efficiency } \\
\cline { 4 - 5 } & treatments & treatments & Absolute content & Relative efficiency \\
\hline Oil extraction rate & $11.5 \%$ & $14.3 \%$ & $2.80 \%$ & $24.34 \%$ \\
Oil extraction production & $3.22 \mathrm{mg}$ & $3.82 \mathrm{mg}$ & $0.60 \mathrm{mg}$ & $18.63 \%$ \\
\hline
\end{tabular}

Table 5

Confirmation experiment for additional formulas with and without ultrasound treatments.

\begin{tabular}{lcccc}
\hline \multirow{2}{*}{ Confirmatory experiment } & Without ultrasound & With ultrasound & \multicolumn{2}{c}{ Raised content and efficiency } \\
\cline { 4 - 5 } & treatments & treatments & Absolute content & Relative efficiency \\
\hline Oil extraction rate & $15.4 \%$ & $18.3 \%$ & $2.90 \%$ & $18.83 \%$ \\
Oil extraction production & $2.77 \mathrm{mg}$ & $3.30 \mathrm{mg}$ & $0.53 \mathrm{mg}$ & $19.13 \%$ \\
\hline
\end{tabular}

ultrasound treatment increased by $0.53 \mathrm{mg}$ and relative efficiency was enhanced $19.13 \%$ (Table 5). Finally, the difference between the prediction and the experiment was evaluated. Oil production by extraction was increased under ultrasound as predicted, with an error of only $0.50 \%$ (Tables 4 and 5). Moreover, the absolute oil extraction rate was close to the prediction, but the relative efficiency was off by $5.51 \%$ possibly because of interactions among factors. 


\section{Conclusions}

In this study, we used Chlorella for oil extraction research and added an exposure to Chlorella's natural ultrasonic frequency and LED lighting with the expectation of increasing the production rate as well as oil content. Our conclusions are summarized as follows.

(1) With oil production being the ultimate consideration, the optimal conditions were found to be an LED intensity of 13000 lux, LED red wavelength $(630 \mathrm{~nm})$, LED cycle of exposure $6 \mathrm{~h} / \mathrm{rest} 6 \mathrm{~h}$, ultrasound frequency of $5 \mathrm{MHz}$, ultrasound intensity of $2.75 \mathrm{~mW} / \mathrm{cm}^{2}$, and ultrasound cycle of exposure $10 \mathrm{~min} /$ rest $10 \mathrm{~min}$.

(2) To maximize experiment oil production, LED intensity was the primary factor, followed by LED cycle, ultrasound cycle, ultrasound frequency, LED wavelength, and ultrasound intensity.

(3) Predictions made using additional formulas reveal that the oil extraction rate increased $2.80 \%$ after an ultrasound exposure of $5 \mathrm{~d}$. Relative efficiency of oil extraction rate and the oil extraction production were enhanced 24.34 and $18.63 \%$, respectively. The confirmation experiment proved that, after an ultrasound exposure of $5 \mathrm{~d}$, the oil extraction rate increased $2.90 \%$, relative efficiency of oil extraction rate increased $18.83 \%$, and oil extraction production increased $19.13 \%$. The error in relative oil extraction efficiency increased about $5.51 \%$, and the error in ralative oil extraction production increased about $0.5 \%$.

\section{References}

1 C. Yusuf: Biotechnol. Adv. 25 (2007) 294.

2 P. Spolaore, C. Joannis-Cassan, E. Duran, and A. Isambert: J. Biosci. Bioeng. 101 (2006) 87.

3 W. T. Coakley and D. Hampton: J. Acoust. Soc. Am. 50 (1971) 1546.

4 P. Rajasekhar, L. Fan, T. Nguyen, and F.A. Roddick: Water Res. 46 (2012) 1473.

5 S.-Y. Hsia and Y.-T. Chou: J. Nanomater. 2014 (2014) Article ID 721267.

6 S.-Y. Hsia and S.-K. Yang: J. Nanomater. 2015 (2015) Article ID 531352.

7 Y. Chisti: Biotechnology. Adv. 13 (2007) 294.

8 Y. Gao, B. Nagy, and X. Liu: J. Supercrit. Fluids. 49 (2009) 345.

9 A. Converti, A. A. Casazza, E. Y. Ortiz, P. Perego, and M. D. Borghi: Chem. Eng. Process. 48 (2009) 1146.

10 M. Greenspan: J. Acoust. Soc. Am. 65 (1979) 608.

11 C. J. Dang, L. W. Schmerr, and A. Sedov: Res. Nondestr. Eval. 14 (2002) 177.

12 S.-Y Hsia, S.-K. Yang, and H.-C. Kuo: Agricultural Wasters-Characteristics, Types and Management, ed. Camille N. Foster (Nova Science Publishers Inc., New York, 2015) Chap. 10. 